\title{
Body Deformation Correction for SPECT Imaging
}

\author{
Songxiang $\mathrm{Gu}^{1}{ }^{1}$, Joseph E. McNamara [Member, IEEE] ${ }^{2}$, Joyeeta Mitra [Member, IEEE] ${ }^{2}$, \\ Howard C. Gifford [Member, IEEE] ${ }^{2}$, Karen Johnson' ${ }^{2}$, Michael A. Gennert [Member, IEEE] ${ }^{3}$, \\ and Michael A. King [Senior Member, IEEE] ${ }^{2}$ \\ ${ }^{1}$ Worcester Polytechnic Institute, Worcester, MA 01609 USA \\ 2 University of Massachusetts Medical School, Worcester, MA 01605 USA \\ ${ }^{3}$ Worcester Polytechnic Institute, Worcester, MA 01609 USA. University of Massachusetts Medical \\ School, Worcester, MA 01605 USA
}

\begin{abstract}
Patient motion degrades the quality of SPECT studies. Body bend and twist are types of patient deformation, which may occur during SPECT imaging, and which has been generally ignored in SPECT motion correction strategies. To correct for these types of motion, we propose a deformation model and its inclusion within an iterative reconstruction algorithm. Two experiments were conducted to investigate the applicability of our model. In the first experiment, the return of the postmotion-compensation locations of markers on the body-surface of a volunteer to approximate their original coordinates is used to examine our method of estimating the parameters of our model and the parameters' use in undoing deformation. The second experiment employed simulated projections of the MCAT phantom formed using an analytical projector which includes attenuation and distance-dependent resolution to investigate applications of our model in reconstruction. We demonstrate in the simulation studies that twist and bend can significantly degrade SPECT image quality visually. Our correction strategy is shown to be able to greatly diminish the degradation seen in the slices, provided the parameters are estimated accurately. We view this work as a first step towards being able to estimate and correct patient deformation based on information obtained from marker tracking data.
\end{abstract}

\section{Index Terms}

Bend; deformation; motion correction; single photon emission computed tomography (SPECT); twist

\section{Introduction}

Single photon emission computed tomography (SPECT) is widely used for assessing relative cardiac perfusion. A SPECT acquisition typically requires about $20 \mathrm{~min}$ for acquisition of the projection images. Patient movement during acquisition can cause misalignment of the projection data. In turn, such misalignment may introduce artifacts into the reconstructed slices. These motion artifacts can impact the diagnostic accuracy of the perfusion images [1]-[6].

Patient motions can be tracked during SPECT imaging using a motion capture system, which is comprised of several cameras and a set of retroreflective body markers [7], [8]. The markers are attached to inelastic but bendable bands about the chest and abdomen of the patient. Currently, we have adopted for clinical usage a commercial system (VICON MX, Oxford, U.K.) because of its flexibility and ease of calibration [9]. Fig. 1 shows a volunteer in the supine imaging position on the table of our SPECT system with 2 bands with attached markers, and 2 of the 5 VICON cameras we employ for motion-tracking. The motion of the separate markers 
can be combined to estimate rigid-body motion (RBM) of the chest, and the vertical motion of markers on the abdomen allows us to track respiration [10]. The estimated RBM can be corrected within iterative reconstruction [11]. The respiratory signal can be used to bin listmode SPECT data acquired in synchrony with motion-tracking for the correction of respiratory motion (RM) [12]-[14]. The human body is not a rigid object, however; there is residual motion even after correction for RBM and RM. In our discussion we will term such nonrigid nonperiodic motion as body deformation. Fig. 2 illustrates an example of body-deformation seen in the residual motion of markers recorded from a patient undergoing cardiac SPECT imaging.

There are many potential types of patient body deformation. In this paper, however, we focus on two kinds of deformation that involve the spine: twist and bend. We define twist as a progressive rotation along the backbone, and bend as a progressive curving of the backbone resulting in it being bent differently than it was initially. Bend can occur in any direction; however a vertical bend upward (sitting up) would be difficult for a patient to maintain for any extended period of time. Thus, lateral bend is the more likely to occur when, for example, the patient wiggles to get comfortable. Without correcting for body deformation, it will be present in the reconstructed slices causing degradation, which could impact the diagnostic accuracy of the study.

This paper presents three steps we have developed for correction of twist and bend: 1) creation of a body model with parameters describing the extent of twist and bend;2) using patient observations in the estimation of the deformation parameters of this model; and 3) image reconstruction employing the model and parameters. Our body model is for imaging in the chest and abdomen region, and is based on a simple mechanical model of the spine within that volume. That is, we assume the spine does not stretch or compress in length but just twists and bends (in addition to any RBM). A set of inherent body variables, such as the spine length and center, are specified to apply the model for each patient. The residual body deformation data remaining after removing RM and RBM from the set of marker motions are processed by the deformation model to estimate the deformation parameters (twist angle, bend angle, and bend direction). Finally, the SPECT projections, the specified deformation model, and the deformation parameters are used in a modified reconstruction algorithm to correct the misalignment caused by the body deformation during acquisition to diminish its impact in the reconstructed slices.

\section{Materials and Methods}

\section{A. Body Modeling}

We define deformations as occurring solely within a region of interest (ROI) (Fig. 3) consisting of a portion of the spine imaged by our SPECT system as defined by the placement of our motion-tracking markers. The deformation happening outside of the ROI is considered as RBM. Usually, the ROI includes the upper thoracic vertebrae. When the patient is lying on a flat imaging table, the spine curve in this region is reduced compared to other postures. Fig. 4 shows three example MRI images of the spines of volunteers illustrating this. These spine shapes are approximately a straight line in the ROI except superiorly due to the support provided for the patient's head, shoulders, and arms, which are raised to be out of the way during SPECT imaging. Therefore, we approximate the spine as a straight line.

Our simple deformation model utilizes 3 parameters: twist angle $(T)$, bend angle $(B)$, and bend direction $(D)$. The twist angle is defined as a rotation of the spine, with the rotation increasing from the pelvis to the head. The bend angle is defined as the extent of curving of the spine (apart from its usual curved shape) along an arc. The bend direction is defined as the angular direction for which the bend occurs with respect to the midsagittal plane. In Figs. 5 and 6, we 
show the effects of twist and bend separately on a patient voxel. We take a voxel $v_{0}$ [Figs. 5 (a) and 6(a)] as the initial position. Fig. 5(b) shows that the initial voxel $v_{0}$ would be moved to $v_{1}$ by twist. Bend can be decomposed into three steps: 1) rotate the body to make the bend upwards in the midsagittal plane; 2) make the body bend in this sagittal plane; and 3) then rotate the body back into the initial position. This process is illustrated in Fig. 6. We rotate voxel $v_{1}$ to $v_{2}$ [Fig. 6(b)]. Bend is applied which moves the voxel from $v_{2}$ to $v_{3}$ [Fig. 6(c)]. Finally, we restore the (newly bent) body to its initial orientation by rotating voxel $v_{3}$ to the final deformed position $v_{4}$ [Fig. 6(d)]. Combining the bend direction and the bend angle, we can model bend motion in any direction (for example, laterally), and not just in the sagittal plane. The order in which the twist and bend operations are performed is arbitrary, but must be consistent throughout the model, as twist and bend do not commute. We have chosen the convention that twist precedes bend.

We acknowledge that this model is a gross simplification of what actually happens to the spine as patients move. The deformation is not necessarily uniform at each joint. Given more information about the spine mechanics one could model the probable fractional change at each vertebra. For example, Vorro [15] evaluated myoelectric data obtained from spinal motion dynamics involved in clinical rotation tests. A functional model is discussed for this myoelectric activity involving a helical spinal motor pattern with a focal area of transition that is dynamic in response to postural and motion demands. To understand human lumbar spine motion, Schwartz [16] performed an in-vivo experiment to develop a method for visualizing the in vivo motion of the entire human lumbar spine region, which is mostly composed of bend motion. However, these studies were made when the patient was sitting. In our case, the patient is lying supine during the SPECT imaging. The relationship between the vertebrae most likely depends on the patient pose. Therefore, in this research, we make the simple assumption that the distributions of bend and twist along the spine are both uniform. This means that each unit length of the spine contributes equally toward the total bend and twist angles. This assumption is an oversimplification, which can be improved upon with refined models in the future.

Other important assumptions for the spine deformation are 1) the spine is incompressible, and 2 ) when the spine is bent, it continues to lie within a single plane. Though not perfectly accurate assumptions, they are good starting points for this research. With these assumptions, we can compute the bend angle and direction directly from the markers attached to the patient.

Based on all the assumptions aforementioned, we build an approximate deformation model of the patient body. If we can quantify the three deformation parameters $T, B$, and $D$, we can map the initial patient body into the deformed position. Such a map can also be expressed as a motion matrix $M$, so that $\vec{f}_{\text {deform }}=\mathbf{M} \vec{f}_{\text {init }}\left(\vec{f}_{\text {init }}\right.$ and $\vec{f}_{\text {deform }}$ are the initial and deformed body volumes).

\section{B. Pattern Independent Deformation Estimation}

For tracking motion, two belts are wrapped about the patient body; one is about the chest and the other about the abdomen, as shown in Fig. 7. Three to seven retroreflective markers are attached to each belt. The pattern of markers on each belt dictates the visibility of the markers during the motion capture procedure, which is crucial to our motion estimation. However, due to the range of torso shapes, there is no one general pattern ideal for all patients. Thus, we place different marker patterns on different patients for motion capture purposes. Fig. 8 shows some examples of marker patterns that are used for motion capture. Because of this potential variability in the pattern of the markers on the belts we have developed a method of estimating our three parameters for body deformation independent of the pattern of markers on the belts.

The chest and abdomen belts have a Velcro layer affixed to them which does not stretch, but does bend. Therefore, the marker patterns on these belts do not change much during the 
deformation. We take the abdomen belt as the baseline for computation in that any motion in that belt after the RM has been removed [10] is taken to be RBM. Using RBM correction, we return the abdomen belt to its initial pose. Then, patient deformation is indicated entirely by the chest belt. Therefore, in the remainder of this paper, we treat the RM and RBM as having been modeled, and henceforth consider the remaining input motion data to comprise only deformation.

Accurately locating the spine is important for two reasons: it provides 1) the center axis for twist (Fig. 5) and 2) the reference line for bend (Fig. 6). Because the spine is oriented axially along the $z$ axis (Fig. 7), it suffices to specify its lateral $x$ axis and vertical $y$ axis positions. We take the spine lateral position as the $x$ coordinate of the centroid of the chest markers. Based on our MRI studies, we approximate the spine vertical position as $1 / 3$ the distance from the surface of the table to the average anterior level of the chest markers (Fig. 4). We conducted a study of the impact of errors in the estimation of the spine location on both deformation estimation and reconstruction, as detailed later in this section.

In our model, each of the belts has an associated key plane that goes through the centroid of the belt markers and intersects the patient spine (Fig. 7). The chest key plane $P_{c}$ and the abdomen key plane $P_{a}$ are determined as the planes perpendicular to the spine which intersect the average axial location of the markers on a given belt. We use these key planes as the start and end of the ROI for deformation measurement. By computing the distance between the two key planes, we estimate the length of a section of spine within the ROI.

We need a patient reference pose for motion correction; all motion computations are carried out with respect to this pose. We pick the initial patient position as the reference, justified by the observation that the ability of patients to remain still decreases over time. The reference pose is determined by the first set of chest marker coordinates $S=\left\{S_{i}\right\}$, where $S_{i}$ is the $i$ th marker's coordinates. After patient motion, the markers have another set of coordinates $S^{\prime}$. Following RM and RBM correction based on the abdominal markers, the chest markers have a third set of coordinates $S^{\prime \prime}$, providing the information needed to determine body deformation.

The affine transformation between $S$ and $S^{\prime \prime}$, which determines the deformation parameters, is given by

$$
S_{i}^{\prime \prime}=s R S_{i}+\tau
$$

where $s$ is a scale factor, $R$ is a rotation matrix, and $\tau$ is a translation vector. Rotation matrix $R$ can be computed [17] as

$$
R=A\left(A^{T} A\right)^{-1 / 2}
$$

where $A=\sum_{i=1}^{n}\left(\left(S_{i}-\bar{S}\right)\left(S_{i}^{\prime \prime}-\bar{S}^{\prime \prime}\right)\right), n$ is the number of markers, $S$ is the average marker location before the patient motion, and $\overline{S^{\prime \prime}}$ is the average marker location after the RM and RBM corrections. As long as the markers are not colinear, matrix $A$ has full rank and matrix $R$ is orthonormal. Scale factor $s$ is taken to be 1 because the marker belts do not stretch. Translation $\tau$ can be found by $\tau=\bar{S}^{\prime \prime}-s R \bar{S}$.

In our model, this rotation is caused by the twist and bend motions. Thus the rotation matrix $R$ can be decomposed into three simple components- twist about the $z$ axis, bend about the $y$ 
axis, and bend direction about the $z$ axis. Considering the movement order defined in Section II-A, we decompose $R$ as

$$
R=R_{D}^{-1} R_{B} R_{D} R_{T}
$$

where

$$
\begin{aligned}
& R_{D}=\left[\begin{array}{ccc}
\cos (D) & -\sin (D) & 0 \\
\sin (D) & \cos (D) & 0 \\
0 & 0 & 1
\end{array}\right] \\
& R_{B}=\left[\begin{array}{ccc}
\cos (B) & 0 & -\sin (B) \\
0 & 1 & 0 \\
\sin (B) & 0 & \cos (B)
\end{array}\right] \\
& R_{T}=\left[\begin{array}{ccc}
\cos (T) & -\sin (T) & 0 \\
\sin (T) & \cos (T) & 0 \\
0 & 0 & 1
\end{array}\right] .
\end{aligned}
$$

$R_{D}$ and $R_{T}$ are rotation matrices about the $z$ axis with bend direction $D$ and twist angle T,

respectively. $R_{B}$ is a rotation matrix about the $y$ axis with bend angle $B$. Note that $R_{D}^{-1}$ is equal to $R_{-D}$ where $D$ is replaced by $-D$. Since the twist and bend directions share the same rotation axis, we can take advantage of

$$
R_{D} R_{T}=R_{D+T}=\left[\begin{array}{ccc}
\cos (D+T) & -\sin (D+T) & 0 \\
\sin (D+T) & \cos (D+T) & 0 \\
0 & 0 & 1
\end{array}\right]
$$

to simplify (3) to [see (4) at the bottom of the page].

$\mid R=R_{-D} R_{B} R_{D+T}=\left[\begin{array}{ccc}\ldots & \ldots & -\sin (B) \cos (D) \\ \ldots & \ldots & \sin (B) \sin (D) \\ \sin (B) \cos (D+T) & -\sin (B) \sin (D+T) & \cos (B)\end{array}\right]$

From the elements of $R$ we can determine the deformation model parameters. Bend angle $B$ can be found from $R_{33}=\cos (B)$; knowing $B$, bend direction $D$ can be found from $R_{13}=-\sin$ (B) $\cos (D)$ or $R_{23}=\sin (B) \sin (D)$; knowing $B$ and $D$, twist angle $T$ can be found from $R_{31}=$ $\sin (B) \cos (D+T)$ or $R_{32}=-\sin (B) \sin (D+T)$. Note, however, that when $B=0, D$ is not uniquely defined. In this case, we take $D=0$ and find $T$ in the usual way.

Equation (3) also illustrates the procedure that we use to model the deformation. We first make a twist movement with the twist angle, rotate the patient body with the bend direction to make the bend occur upward in the sagittal plane, bend the patient body with the bend angle, and then rotate the body back to counteract the first rotation. In this way our model can deal with bend in any direction and not just in the sagittal plane.

The motion modeled in (1) is an affine transformation between the initial marker locations for the chest belt and that of the chest belt after RM and RBM correction. The twist angle $T$ and bend angle $B$ computed in this way are the total twist and bend within the ROI, that is, from 
key plane $P_{a}$ to key plane $P_{c}$. Deformation at intermediate locations in the ROI volume is modeled as the incremental decrease in $B$ and $T$ to zero as one proceeds axially from the chest end of the ROI to the abdominal end as illustrated in Fig. 9. We call these intermediate deformation values the local twist and bend angles. Note that the bend direction $D$ keeps the same value for all voxels due to the assumption that the spine continues to lie within a single plane.

To estimate the local twist and bend angles for a specific voxel within the ROI, we rely on the assumption that the angles increase linearly along the spine. For a specific voxel $v$ the local twist angle is $T_{v}=T L_{v} / L_{S}$, where $L_{S}$ is the spine length within the ROI and $L_{v}$ is the distance between voxel $v$ and the abdomen key plane $P_{a}$. Similarly, the local bend angle $B_{v}$ is $B_{v}=$ $B L_{v} / L_{s}$. Combined with the deformation description in Section II-A, we can estimate the movement of each voxel within the ROI using the local deformation parameters.

\section{Deformation Matrix}

The deformation matrix is a mapping connecting the initial body with the deformed body specifying the deformation at each voxel of the body volume. It is a realization of the simple spine model, parameterized by $B, D$, and $T$ and the location of the spine. This mapping can be described as a deformation matrix $M$ that may change as a function of time during an acquisition. The mapping is given as

$$
\vec{f}_{\text {deform }}=M(t) \vec{f}_{\text {init }}
$$

where $\vec{f}_{\text {init }}$ and $\vec{f}_{\text {deform }}$ are the reference (initial) and deformed reconstruction volumes, treated as column vectors. We can then write the acquired projection data $\vec{g}$ as a column vector

$$
\begin{aligned}
& \vec{g}=\left[\begin{array}{c}
\vec{g}_{0} \\
\vec{g}_{1} \\
\ldots \\
\vec{g}_{N-1}
\end{array}\right] \\
& =\left[\begin{array}{cccc}
A_{0} & 0 & \ldots & 0 \\
0 & A_{1} & \ldots & 0 \\
\ldots & \ldots & \ldots & \ldots \\
0 & 0 & \ldots & A_{N-1}
\end{array}\right]\left[\begin{array}{c}
M_{0} \\
M_{1} \\
\ldots \\
M_{N-1}
\end{array}\right] \vec{f}_{\text {init }}
\end{aligned}
$$

where $N$ is the number of acquired projections; $\vec{g}_{i}$ is the sub-vector of $\vec{g}$ corresponding to the $i$ th projection state; $A_{i}$ is the submatrix of the system matrix $A$ related to $\vec{g}_{i}$ which includes imaging geometry, attenuation, scatter, and distance-dependent spatial resolution; and $M_{i}=\int_{t=T_{i}}^{T_{i+1}} M(t) d t$ where $T_{i}$ is the start time of the $i t h$ projection and $M(t)$ is the deformation matrix at time $t$. For time intervals where the patient has not moved or deformed $M_{i}$ is the identity matrix. Note that (6) is exact and reduces, in the motion-free case, to the familiar

$$
\vec{g}=A \vec{f}=\left[\begin{array}{c}
A_{0} \\
A_{1} \\
\ldots \\
A_{N-1}
\end{array}\right] \vec{f}
$$




\section{Modified Reconstruction Algorithm}

A number of investigators have investigated including motion into maximum likelihood expectation maximization (MLEM) based iterative reconstruction [11], [18]-[23]. We adapt the formalism put forth by Feng et. al. [11] modified to account for body deformation as well as RBM. The system matrix $A$ with elements $a_{i j}$ is replaced by $A M$ with elements $\Sigma_{\boldsymbol{k}} a_{i k} m_{k j}$. We write this modified MLEM reconstruction equation as

$$
f_{j}^{\text {new }}=\frac{f_{j}^{\text {old }}}{\sum_{k} m_{j k}^{T} \sum_{i} a_{k i}^{T}} \sum_{k} m_{j k}^{T} \sum_{i} a_{k i}^{T} \frac{g_{i}}{\sum_{k^{\prime}} a_{i k} \sum_{j^{\prime}}\left(m_{k^{\prime} j_{j}} f_{j}^{\text {old }}\right)}
$$

where $f_{j}^{\text {new }}$ and $f_{j}^{\text {old }}$ are the update and previous values of voxels $j$ in the reconstruction volume, $a_{i j}$ are elements of the system matrix $A, g_{i}$ are the $2 \mathrm{D}$ projection bin values, and $m_{k j}=m_{j k}^{T}$ are elements of $M_{i}$ at the time corresponding to acquisition $g_{i}$.

Note that $A$ includes attenuation and scatter. As in [11] we assume we have an attenuation map for the patient in the initial position. Note that the attenuation map and scatter estimate should be modified to match the patient RM, RBM, and body deformation. To simplify this process one can employ the triple energy window (TEW) scatter estimation method [24]. In this method scatter is estimated from scatter windows acquired simultaneously with the photopeak data. Thus, the scatter estimate naturally matches the motion state of the patient. To avoid the issue of negative values when the scatter estimate is subtracted from the acquired data one can follow the methodology of [25] and add the scatter estimation to the scatter-free projection data.

An important concept in the modified MLEM update equation is back-deformation, which plays a role similar to backprojection. In traditional MLEM, the operation $\sum_{i} a_{j i}^{T} g_{i}$ is the backprojection of projection data $g_{i}$ as determined by the system matrix into the reconstruction volume, arising as an alternative to inverting system matrix $A$ directly, which is at best an illposed problem. Analogously, in our modified MLEM algorithm, the operation

$\sum_{k} m_{j k} \sum_{i} a_{k i}^{T} g_{i}$ is the back-deformation of deformed volume $\sum_{i} a_{k i}^{T} g_{i}$ as determined by the deformation matrix into the back-deformed volume, arising as an alternative to inverting deformation matrices $M_{i}$; directly, which may also be an ill-posed problem.

\section{Experimental Procedure}

We now describe several experiments that validate our deformation model and its application to motion compensation.

\section{A. Correction of Motion of Markers on a Volunteer}

Returning now to motion detection, the surface markers that we have detected may be considered as a set of special voxels during the computation. We can back-deform the deformed surface markers to investigate the correctness of our back-deformation computation. Ideally, the back-deformed markers should be returned exactly to their initial positions. However, due to the inaccuracy of the motion estimation procedure, there will always be some error in the back-deformation, leading to some discrepancy between these two results.

We performed an experiment to determine the extent of this discrepancy. For this experiment, a volunteer was outfitted with two belts with five markers each. The volunteer was instructed 
to make a series of deformations while lying on the imaging bed of the SPECT system. Optical cameras tracked the marker motion at a frame rate of 30 frames/sec. We first estimated and then applied RM and RBM corrections to make the abdomen belt stationary. The residual motion on the chest belt was considered as the input data for the body deformation computation. We then estimated body deformation as described earlier. Next, we applied a backdeformation operation to the chest markers to correct for the deformation. The deformation correction operation is the inverse of the deformation process. This backdeformation operation uses $-T$, $-B$, and $D$ as the twist, bend, and bend direction to apply to the deformed markers. We expect that the markers should be reset close to their initial positions, with little or no residual marker motion. Thus, as an error measure we calculated the root-mean-square error (RMSE)

$\operatorname{RMSE}_{\text {motion }}$ for the $x, y$, and $z$ components of the residual marker motions for each chest marker as shown in (9) at the bottom of the page.

$$
\text { RMSE }_{\text {motion }}=\sqrt{\frac{\sum_{i=0}^{L} \sum_{j=0}^{K}\left[\left(x_{i j}-x_{i 0}\right)^{2}+\left(y_{i j}-y_{i 0}\right)^{2}+\left(z_{i j}-z_{i 0}\right)^{2}\right]}{L K}} .
$$

where $\left(x_{i j}, y_{i j}, z_{i j}\right)$ is the $i$ th marker's position in observation $j,\left(x_{i 0}, y_{i 0}, z_{i 0}\right)$ is the marker's reference position, $L$ is the number of chest markers, and $K$ is the number of observations.

The spine position in the $x y$ plane is a crucial parameter for our motion estimation model. If available one can estimate the spine location from registered CT or MRI slices; however, this is not always available. Such was the case for the volunteer in this study. To measure the effect of errors caused by spine location misestimation, we investigated the effect of varying the spine location used in our deformation correction. The estimated locations varied from $-50 \mathrm{~mm}$ to $+45 \mathrm{~mm}$ in the $x$ axis and from -65 to +60 for the $y$ axis in $5 \mathrm{~mm}$ steps about our selected location. A total of 520 spine locations sampled in this way.

\section{B. Test of Deformation Correction Using Simulated SPECT Acquisitions}

To test our method of deformation correction, we created source and attenuation distributions for a simulated Tc-99m combined cardiac and bone imaging agent distribution using the mathematical cardiac torso (MCAT) anthropomorphic phantom [26], [27]. The simulated Tc-99m distribution was created to enable the visualization of the heart, which is the target of our clinical research effort and the spine, which is the focus of our deformation model. The source and attenuation distributions data volumes were $256 \times 256 \times 256$ with a pixel size of $0.208 \mathrm{~cm}$. We performed a simulation with twist angle $T=15^{\circ}$, bend angle $B=25^{\circ}$, and bend direction $D=15^{\circ}$. The position of the spine was taken as the location of the center of the spinal canal.

An analytical projector, which included attenuation and distance-dependent spatial resolution, was used to create the projections. We did not include scatter in this simulation. We modeled acquisition as occurring simultaneously from three gamma camera heads spaced at 120 degrees to simulate the acquisition of bone SPECT on a Philips Irix SPECT system. Acquisition consisted of 120 projections total with 40 acquired by each head. Therefore, any motion would have affected 3 projections simultaneously. The attenuation map was deformed corresponding to the deformation of the MCAT source distribution.

Once the projection sets were created, projections $P_{\text {int }}$ from the original motion volume and projections $P_{\text {deform }}$ from the deformed volume were mixed to simulate a simple patient deformation occurring halfway through the acquisition. That is, the new projection file $P_{\text {mix }}$ 
consisted of 120 projections, 60 projections from $P_{\text {init }}(1-20,41-60,81-100)$ and 60 projections from $P_{\text {deform }}(21-40,61-80,101-120)$.

The projections sets $P_{\text {init }}$ and $P_{\text {mix }}$ were reconstructed by 24 iterations of MLEM using (8). MLEM reconstruction included attenuation correction. The attenuation map employed when deformation was present was that of the MCAT attenuation distribution deformed using the motion model employed within reconstruction. Thus, for the case of body deformation with the level of the spine chosen to be the same as that used in the simulation, there is an exact match between the attenuation maps used in simulation and reconstruction. With correction of deformation using a level of the spine other than the correct one, there will be a mismatch between the maps used in simulation and reconstruction. Scatter correction was not included as scatter was not included in forming the projections.

The slices reconstructed from the projection set created without motion $P_{\text {init }}$ were taken as the standard against which the reconstructions of $P_{\text {mix }}$ were compared. We performed several comparisons: 1) no motion compensation, 2) motion compensation using the correct spine location, and 3) motion compensation with inaccurate spine locations. To quantitatively

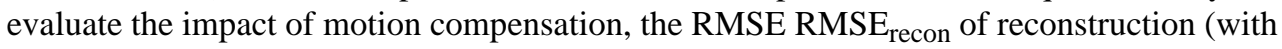
and without motion compensation) was calculated by

$$
\text { RMSE }_{\text {recon }}=\sqrt{\sum_{i \in R O I}\left(\text { counts }_{i}-\text { true }_{-} \text {counts }_{i}\right)^{2}}
$$

where $N$ is the number of voxels inside the $R O I$, counts ${ }_{i}$ are the counts in the slices reconstructed from $P_{\text {mix }}$ for voxel $i$, and true_counts ${ }_{i}$ are the counts in the slices reconstructed from $P_{\text {init }}$ for voxel $i$.

Again, the spine position in the $x y$ plane is a parameter, which must be estimated to specify the twist axis and the initial position of the spine for application of the bend. With the MCAT simulations we know this parameter exactly. In clinical situations, we will not know the spine position exactly. Thus, we performed a study of the effect of varying the spine location in 5 $\mathrm{mm}$ steps about the actual spine position. The 49 grid positions covered a region from -15 to $+15 \mathrm{~mm}$ to either side of the actual spine location for both the $x$ and $y$ directions. For each grid

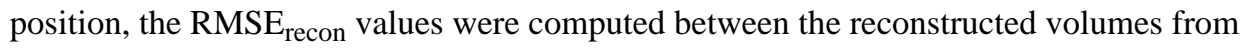
$P_{\text {mix }}$ and $P_{\text {init }}$ as described above.

\section{Results}

\section{A. Correction of Motion of Markers on a Volunteer}

Fig. 10(a) shows the $x, y$, and $z$ components of the tracked location of the center marker on the chest of a volunteer. Note that respiratory motion has been corrected before our processing [10]. We estimated the RBM based on the motion of the abdominal markers with the goal of making their locations stationary as required by our deformation model. After we acquired the RBM motion from the abdomen markers, we applied this RBM correction to the chest markers to estimate the chest marker's motion after the abdomen markers have been realigned to their initial positions [Fig. 10(b)]. Thus, any remaining motion of the chest marker's indicates body deformation. We calculated the deformation parameters with the spine model as described above and applied deformation correction. The result, shown in Fig. 10(c), is a large decrease of the marker's motion in the $x$ direction and moderate decreases of the marker's motion in the other 2 directions. 
A comparison of the RMSE $E_{\text {motion }}$ values for the five chest markers is shown in Fig. 11. We see that RBM correction reduced the RMSE $E_{\text {motion }}$ value for some markers, while increasing it

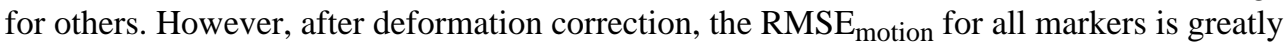
decreased.

The results of our investigation of the sensitivity of deformation correction to the location of

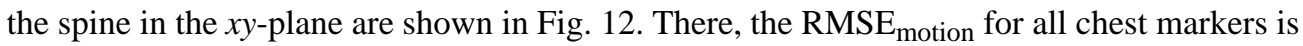
graphed for each of the 520 locations in the grid of positions about that originally estimated for the spine. The values of the RMSE $\mathrm{E}_{\text {motion }}$ vary from 9.65 to 14.34 . The minimum $\mathrm{RMSE}_{\text {motion }}$ value 9.65 is not far away from the RMSE $\mathrm{m}_{\text {motion }}$ value of the originally estimated spine location, which is 9.81 . Therefore, the error caused by the spine location for the deformation parameter estimation is not significant.

\section{B. Test of Deformation Correction Using Simulated SPECT Acquisitions}

The results of applying deformation correction to SPECT simulations are illustrated in Fig. 13, which shows transverse slices through the heart region of the MCAT phantom. Fig. 13(a) is a slice from the reconstruction when there was no motion in the projections; Fig. 13(b) is a slice for when same deformation was included in half of the projections without deformation correction in the reconstruction, and Fig. 13(c) is a slice for when same deformation was included in half of the projections and the deformation correction was included in reconstruction. Reconstruction was via 24 iterations of MLEM, which included attenuation correction, but no scatter correction. Notice the significant degradation caused throughout the slice by the deformation and how well deformation correction returned the distribution to its original appearance when deformation correction was applied. Numerically, deformation correction reduced the $\mathrm{RMSE}_{\text {recon }}$ from $4.1 \times 10^{-2}$ with no correction to $3.4 \times 10^{-3}$ with deformation correction, thus, verifying the visual impression of a considerable improvement with deformation correction. Fig. 14 shows coronal slices illustrating the impact of deformation and its correction on the spine, ribs, liver, spleen, and kidneys. Note the obvious bend of the spine laterally and its correction. Also note that the kidneys are not impacted by the bend or its correction as they were outside the ROI where it occurred.

Fig. 15 shows the results of our study of the sensitivity of deformation correction to the identification of the spine location. The $\mathrm{RMSE}_{\text {recon }}$ values increase quickly as the magnitude of error increases. The values vary from $3.4 \times 10^{-3}$ to $2.1 \times 10^{-1}$ in the grid of positions about the actual location. Therefore, it appears that our methodology is sensitive to the spine location parameter used in deformation correction and that correction would work best when there are registered CT or MRI slices available to provide an accurate estimate of this location.

\section{Discussion}

Our research team has put significant effort into investigation of respiratory and rigid-body motion, but this is our first step into nonrigid motion estimation and correction [7]-[11], [13]. It is motivated by our observation that after extracting respiratory and rigid-body motion components from multiple marker tracking data for patients we find cases where there is a residual motion which does not appear to be respiratory motion related. This is illustrated in Fig. 2 where the chest and abdomen markers show different extents of rotation about the body axis (twist) and change in the angulation of the planes associated with the belts (bend). Based on these observations we have started exploration of the detection and correction of these forms of deformation as our first step into nonrigid motion correction.

We have developed a model for body deformation and methods for estimating its parameters. We have successfully applied this model to correct the motion of volunteers. We have also 
applied the model to correct body deformation in a simulation study with excellent results so long as all the deformation parameters are known relatively accurately.

Future studies will include an extensive investigation of the frequency of occurrence of nonrigid body motion, its impact, and whether we can successfully separate it from respiratory motion and rigid-body patient motion in a large set of clinical acquisitions. We also anticipate conducting an MRI-based experiment to provide an anatomical assessment of our methods.

Motion can cause parts of the body to move into and out of the field-of-view (FOV) of the camera heads. This happens every time there is axial motion with cardiac imaging, and can occur within the transverse plane especially for larger patients and small FOV cardiacdedicated SPECT systems. Since the heart is typically imaged within the central portion of the FOV it is unlikely that such motion would lead to truncation of the heart in the projections. Thus, this should not be a significant problem for visualizing the heart in cardiac SPECT. It could be a problem with portions of the slices away from the heart and in general SPECT imaging. Care must be taken when implementing motion correction to pad the reconstruction volume such that regions of the body seen for some projection angles but not others are not lost when they move out of and then back into the reconstruction volume.

Our model is limited in the extent of the deformations we have included, and by the number of assumptions we have employed to implement estimation and correction of deformation within it. Relaxing assumptions such as the uniform progression of twist and bend of the spine within the ROI would be a place to improve upon this first step. Accounting for the biomechanical properties of the other tissues of the body, so that we consider more than just the spine, would also be a potential place to improve upon the start proposed herein. One possible way to achieve such refinement would be to implement a finite-element model (FEM) of the chest and upper abdomen. FEM analysis of this region has been performed by others [28]-[30]. We propose that such modeling be based on CT slices acquired with SPECT/CT (and PET/CT). The motion tracking could then serve to provide boundary condition information to the model such that the motion of the internal structures would be better estimated than with our current model.

Separation of marker motion into information that can correct RBM, RM, and body deformation is still an open topic. We have made progress in separating RBM from the signal used to estimate RM [13]. However much remains to be done especially for body deformation, which we are just starting to address. Respiratory motion correction usually involves individual markers only [10]. RBM correction takes all the markers as one object and ignores deformation. Our body deformation model considers each belt as one object and estimates the body deformation by the relative movement between the chest belt and abdomen belt. However our method depends on the successful removal of RBM and any influence of RM from the marker data. Thus imperfections in the first two impact the accuracy of estimation of body deformation.

\section{Conclusion and Future Work}

In this paper, we presented a body deformation model, defined by body twist, body bend, and bend direction. With this model, we formulated a methodology for estimating a set of parameters to describe the modeled patient deformation. A set of volunteer motion data was acquired to investigate the use of our model and the parameters derived from it to correct marker data for body deformation. We also investigated use of a deformation matrix based on our model in MLEM correction of deformation, and investigated how sensitive our model was to the estimation of the spine location. Compared to the reconstruction volume with deformation but no compensation, our compensated reconstruction volume shows a significant improvement. However, our correction was determined to be sensitive to the estimation of the 
spine location. We, thus, view this work as but a first step towards being able to estimate and correct patient deformation based on information obtained from marker tracking data.

\section{Acknowledgments}

The contents are solely the responsibility of the authors and do not necessarily represent the official views of the NIBIB. The material of this manuscript was published in part as S. Gu, J. E. McNamara, J. Mitra, H. C. Gifford, K. Johnson, M. A. Gennert, and M. A. King, "Body deformation correction for SPECT imaging," in Proc. 2007 IEEE Nucl. Sci. Symp. Med. Imag. Conf. [31], and as S. Gu, J. E. McNamara, K. Johnson, H. Gifford, A. V. Sklyar, M. A. Gennert, and M. A. King, "Pattern independent deformation estimation illustrated by MRI," in Proc. 2008 IEEE Nucl. Sci. Symp. Med. Imag. Conf. [32].

This work was supported by the National Institute of Biomedical Imaging and Bioengineering by Grant R01EB001457.

\section{References}

1. Botvinick EH, Zhu YY, O'Connell WJ, Dae MW. A quantitative assessment of patient motion and its effect on myocardial perfusion SPECT images. J Nucl Med 1993;34(2):303-310. [PubMed: 8429354]

2. Prigent FM, Hyun M, Berman DS, Rozanski A. Effect of motion on thallium-201 SPECT studies: A simulation and clinical study. J Nucl Med 1993;34(11):1845-1850. [PubMed: 8229222]

3. Cooper JA, Neumann PH, McCandless BK. Effect of patient motion on tomographic myocardial perfusion imaging. J Nucl Med 1992;33(8):1566-1571. [PubMed: 1634955]

4. Reutter BW, Lim S, Huesman RH, Coxson PG, Klein GJ, Budinger TF. Cardiac creep during rest/ stress myocardial perfusion studies C patient motion and lung air redistribution. J Nucl Med 1996;37 (5):131-131.

5. Segars WP, Tsui BMW. Study of the efficacy of respirator gating in myocardial SPECT using the new 4D NCAT phantom. IEEE Trans Nucl Sci 2002;49:675-679.

6. Livieratos L, Rajappan K, Stegger L, Schafers K, Bailey DL, Camici PG. Respiratory gating of cardiac PET data in list-mode acquisition. Eur J Nucl Med Mol Imag 2006;33(5):584-588.

7. Bruyant PP, King MA, Pretorius PH. Correction of the respiratory motion of the heart by tracking of the center of mass of thresholded projections: A simulation study using the dynamic MCAT phantom. IEEE Trans Nucl Sci 2002;49:2159-2166.

8. Bruyant PP, Gennert MA, Speckert GC, Beach RD, Morgenstern JD, Kumar N, Nadella S, King MA. A robust visual tracking system for patient motion detection in SPECT: Hardware solutions. IEEE Trans Nucl Sci 2005;52:1288-1294. [PubMed: 19081772]

9. McNamara JE, Pretorius H, Johnson K, Mitra J, Dey J, Gennert MA, King MA. A flexible multicamera visual-tracking system for detecting and correcting motion-induced artifacts in cardiac SPECT slices. J Med Phys to be published.

10. Mukherjee JM, McNamara JE, Johnson KL, Dey J, King MA. Estimation of rigid-body and respiratory motion of the heart from marker tracking data for SPECT motion correction. IEEE Trans Nucl Sci 2009;56:147-155.

11. Feng B, Gifford HC, Beach RD, Boening G, Gennert MA, King MA. Use of three-dimensional gaussian interpolation in the projector/backprojector pair of iterative reconstruction for compensation of known rigid-body motion in SPECT. IEEE Trans Med Imag 2006;25:838-844.

12. Ue, H.; Haneishi, H.; Iwanaga, H.; Suga, K. Motion correction of respiratory-gated SPECT image and clinical usefulness of the extracted motion information. Conf Rec 2005 IEEE Nucl Sci Symp; 2005. p. 2418-2422.

13. Dey, J.; Feng, B.; Johnson, KL.; McNamara, JE.; Pretorius, PH.; King, MA. Respiratory motion correction in cardiac SPECT using affine and free-form deformation registration with temporal and spatial constraints. Proc. 2007 Fully Three-Dimens. Reconstruc. Conf; 2007. p. 201-204.

14. Kovalski G, Israel O, Keidar Z, Frenkel A, Sachs J, Azhari H. Correction of heart motion due to respiration in clinical myocardial perfusion SPECT scans using respiratory gating. J Nucl Med 2007;48(4):630-636. [PubMed: 17401102] 
15. Vorro J, Johnston WL. A myoelectric model for thoracic spinal motion dynamics during clinical rotation tests: Part 2. bilateral segmental motor behaviors. J Amer Osteopath Assoc 2003;103(5): 232-238. [PubMed: 12776764]

16. Schwartz, MH.; Rozumalski, A.; Wervey, R.; Novacheck, TF.; Swanson, A.; Dykes, DC. Visualizing the in vivo three-dimensional motion of the human lumbar spine: A novel technique. Proc. 12th Ann. Gait and Clinical Movement Analysis Soc; 2007.

17. Horn BKP, Hilden HM, Negahdaripour S. Closed-form solution of absolute orientation using orthonormal matrices. Opt Soc Amer 1988;5:1127-1135.

18. Fulton RR, Hutton BF, Braun M, Ardekani B, Larkin RS. Use of 3-D reconstruction to correct for patient motion in SPECT. J Phys Med Biol 1994;39:563-574.

19. Hutton BF, Kyme AZ, Lau YH, Skerrett DW, Fulton RR. A hybrid 3-D reconstruction/registration algorithm for correction of head motion in emission tomography. IEEE Trans Nucl Sci 2002;49:188194.

20. Klein GJ, et al. Four-dimensional affine registration models for respiratory-gated PET. IEEE Trans Nucl Sci 2001;48:756-760.

21. Schumacher $\mathrm{H}$, et al. A new and flexible reconstruction framework for motion correction in SPECT imaging. IEEE Trans Nucl Sci 2007;54:480-485.

22. Lamare F, Carbayo MJL, Cresson T, Kontaxakis G, Santos A, Rest CCL, Reader AJ, Visvikis D. List-mode-based reconstruction for respiratory motion correction in PET using ono-rigid body transformations. J Phys Med Biol 2007;52(8):5187-5204.

23. Dey J, King MA. Theoretical and numerical study of an MLEM and OSEM reconstruction algorithms for motion correction in emission tomography. IEEE Trans Nucl Sci. submitted for publication.

24. Ogawa K, Harata Y, Ichihara T, Kubo A, Hashimoto S. A practical method for position-dependent compton-scatter correction in single photon emission CT. IEEE Trans Med Imag 1991;10:408-412.

25. King MA, deVries DJ, Pan TS, Pretorius PH, Case JA. An investigation of the filtering of TEW scatter estimates used to compensate for scatter with ordered subset reconstructions. IEEE Trans Nucl Sci 1997;44(3):1140-1145.

26. Tsui BMW, Zhao XD, Gregoriou GK, Lalush DS, Frey EC, Johnson RE, McCartney. Quantitative cardiac SPECT reconstruction with reduced image degradation due to patient anatomy. IEEE Trans Nucl Sci 1994;41:2838-2844.

27. Pretorius PH, King MA, Tsui BMW, LaCroix KJ, Xia W. A mathematical model of motion of the heart for use in generating source and attenuation maps for simulating emission imaging. J Med Phys 1999;26:2323-2332.

28. Sundaram SH, Feng CC. Finite element analysis of the human thorax. J Biomechan 1977;10:505516.

29. Kimpara H, Lee JB, Yang KH. Development of a three-dimensional finite element chest model for the 5th percentile female. J Stapp Car Crash 2005;49:251-269.

30. Merkle AC, Ward EE, O'Connor JV, Roberts JC. Assessing behind armor blunt trauma (BABT) under NIJ standard-0101.04 conditions using human torso models. J Trauma 2008;64:1555-1561. [PubMed: 18545123]

31. Gu, S.; McNamara, JE.; Mitra, J.; Gifford, HC.; Johnson, K.; Gennert, MA.; King, MA. Body deformation correction for SPECT imaging. Proc. IEEE Nucl. Sci. Symp. Med. Imag. Conf; 2007. p. 2708-2714.

32. Gu, S.; McNamara, JE.; Mitra, J.; Johnson, K.; Gifford, H.; Skylar, AV.; Gennert, MA.; King, MA. Pattern independent deformation estimation illustrated by MRI. Proc. IEEE Nucl. Sci. Symp. Med. Imag. Conf; 2008. p. 5285-5291. 


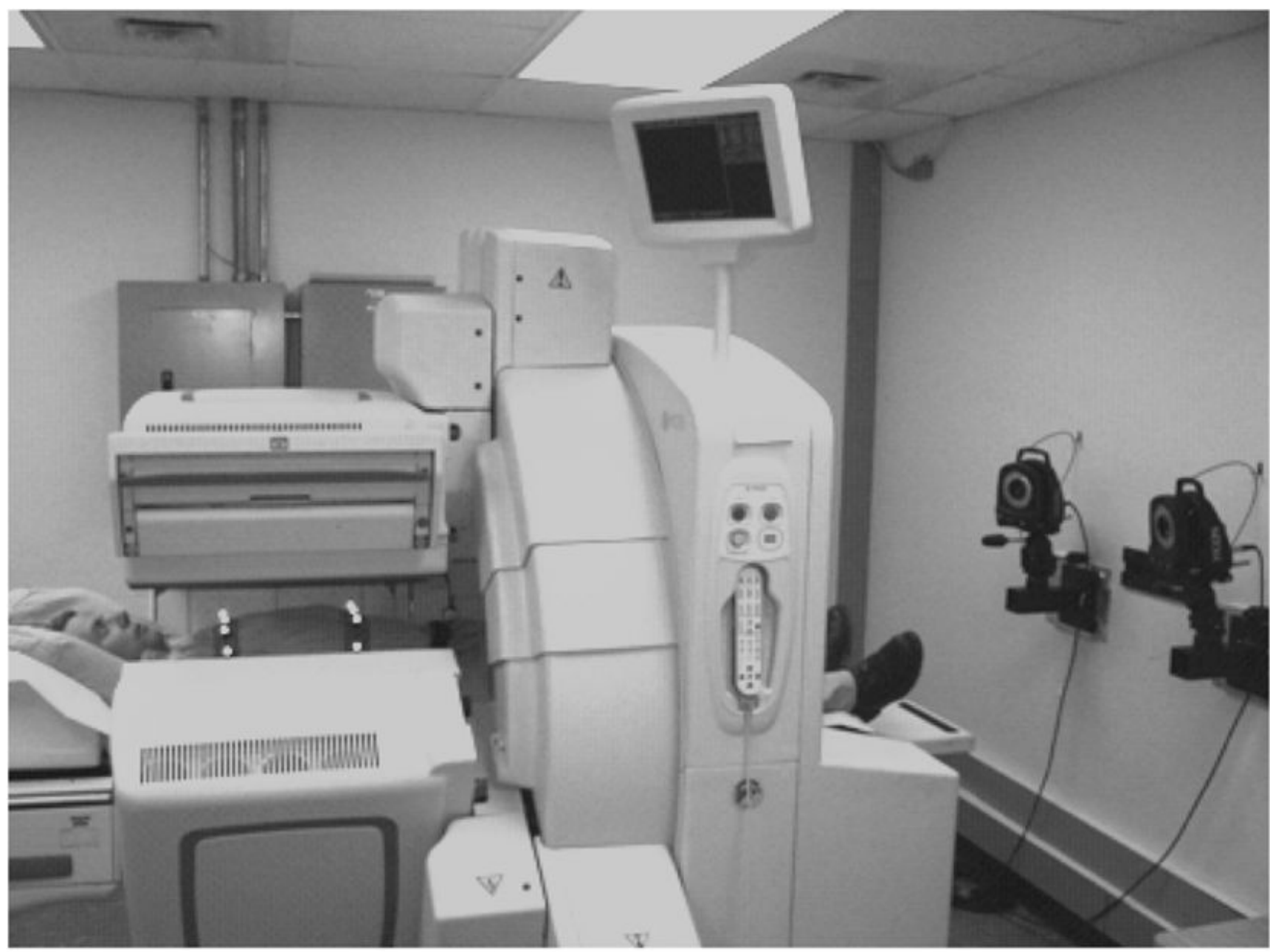

Fig. 1.

Illustration of viewing geometry for markers on stretchy bands and a volunteer in the supine position for motion tracking during SPECT imaging. 


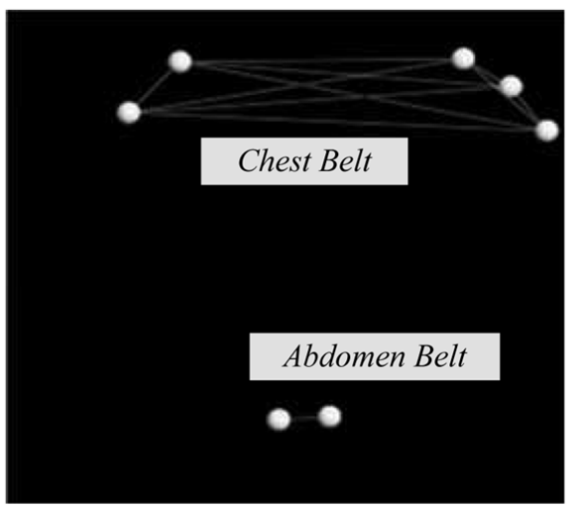

(a)

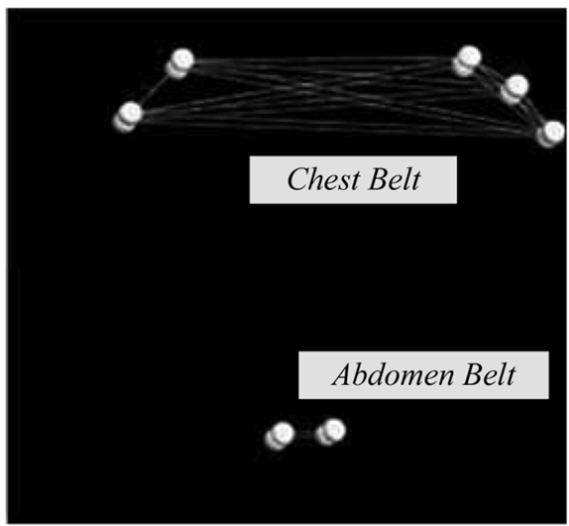

(b)

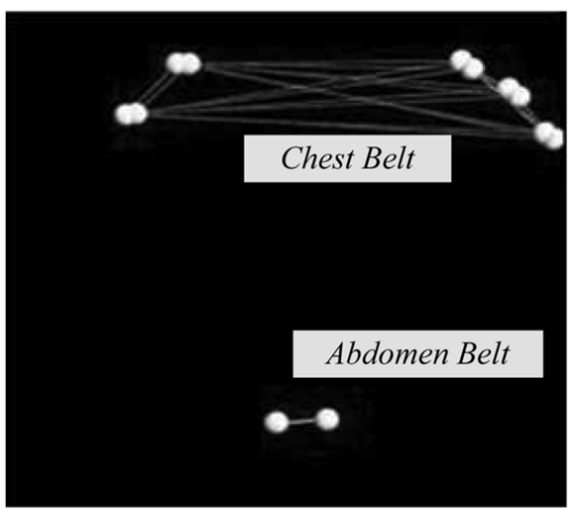

(c)

Fig. 2.

Shown as spheres are the coronal view locations of markers on the abdomen and chest belts of a patient during clinical cardiac SPECT imaging with motion tracking. (a) Initial marker positions. (b) Initial and post-deformation marker locations (darker spheres). (c) Initial and postdeformation locations after RBM correction of abdominal sphere locations to match their initial positions (darker spheres) as per the first step in the correction methodology proposed herein. Note residual marker motion is a combined clockwise twist when viewed from the direction of the patient's feet and bend to the left in 3D, and only the projection of this motion as viewed coronally is presented in this figure. 


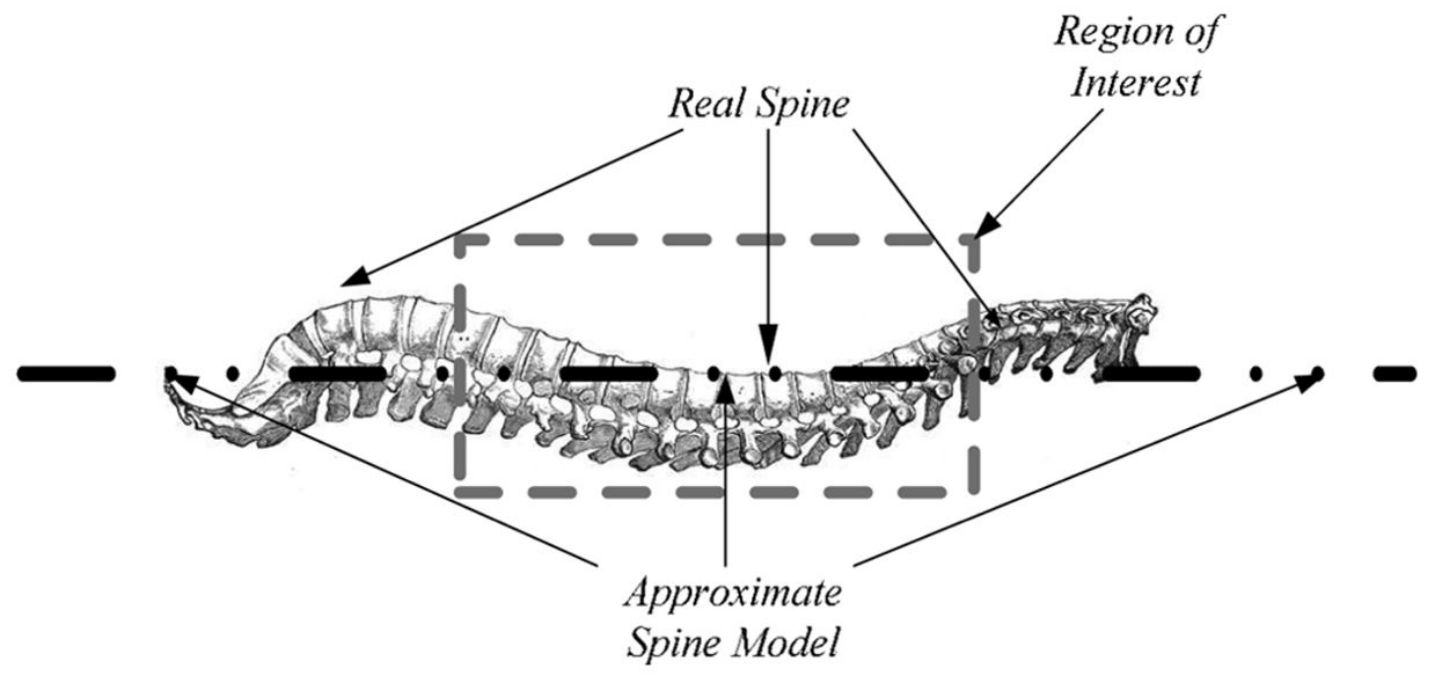

Fig. 3.

Approximate spine model. 

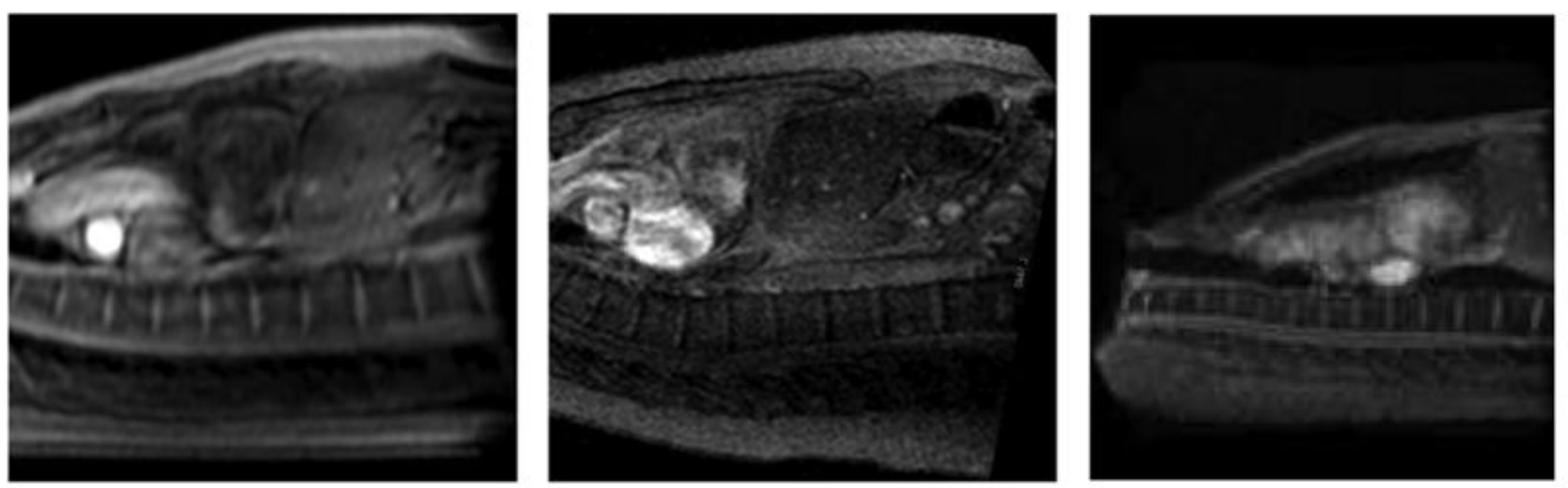

Fig. 4.

Three examples of MRI images showing the spine in the ROI. 


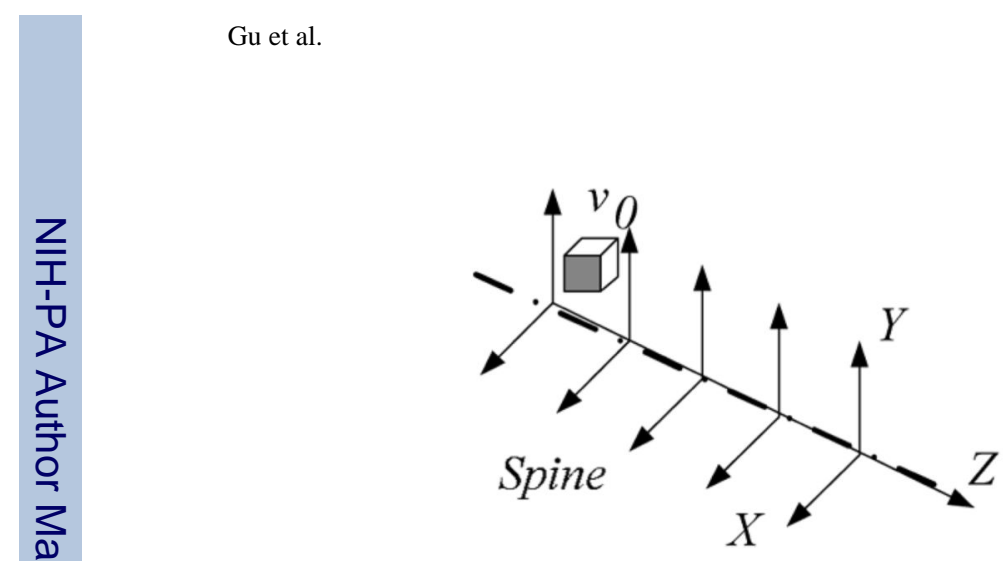

(a)

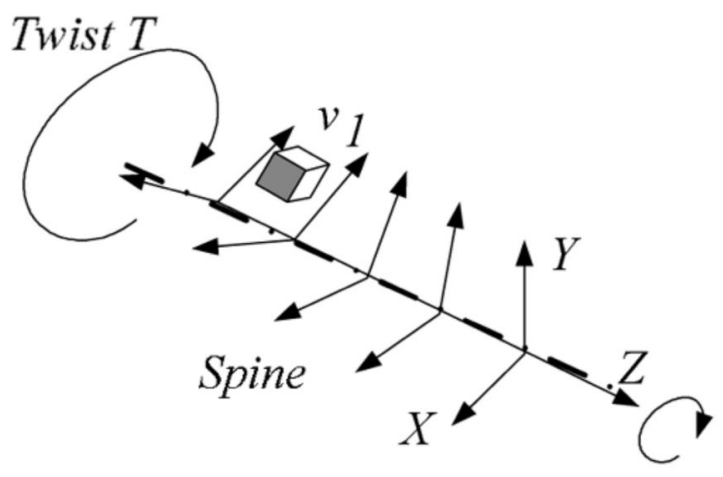

(b)

Fig. 5.

Twist Model. (a) Initial position. (b) Progressive body twist about the spine, which by our convention increases from the abdomen to the chest. 


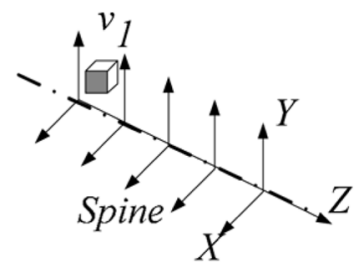

(a)

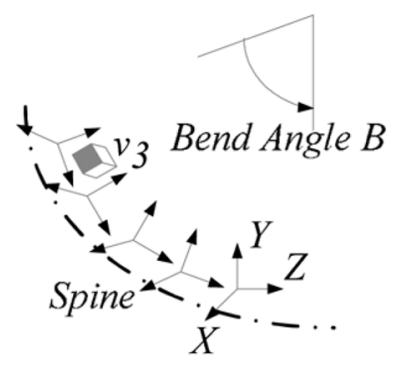

(c)
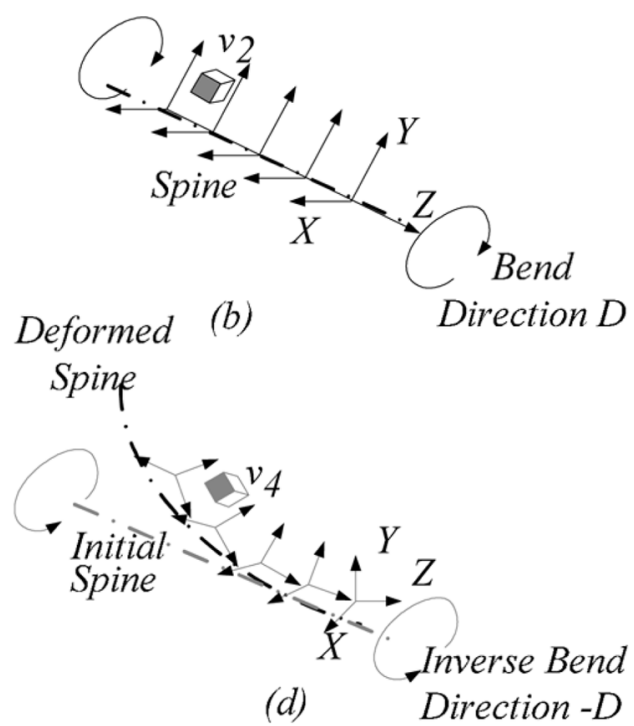

Fig. 6.

Bend Model. (a) Initial position. (b) Rotate the body about the spine by an angle equal to the bend direction $D$ so that we can apply bend in the sagittal plane. (c) Bend the body by the bend angle amount. (d) Rotate the body back to its original orientation with respect to the spine by application of the inverse of the bend direction $-D$. The net effect is a bending by amount Bend Angle $B$ in the plane defined by the Bend Direction $D$. 


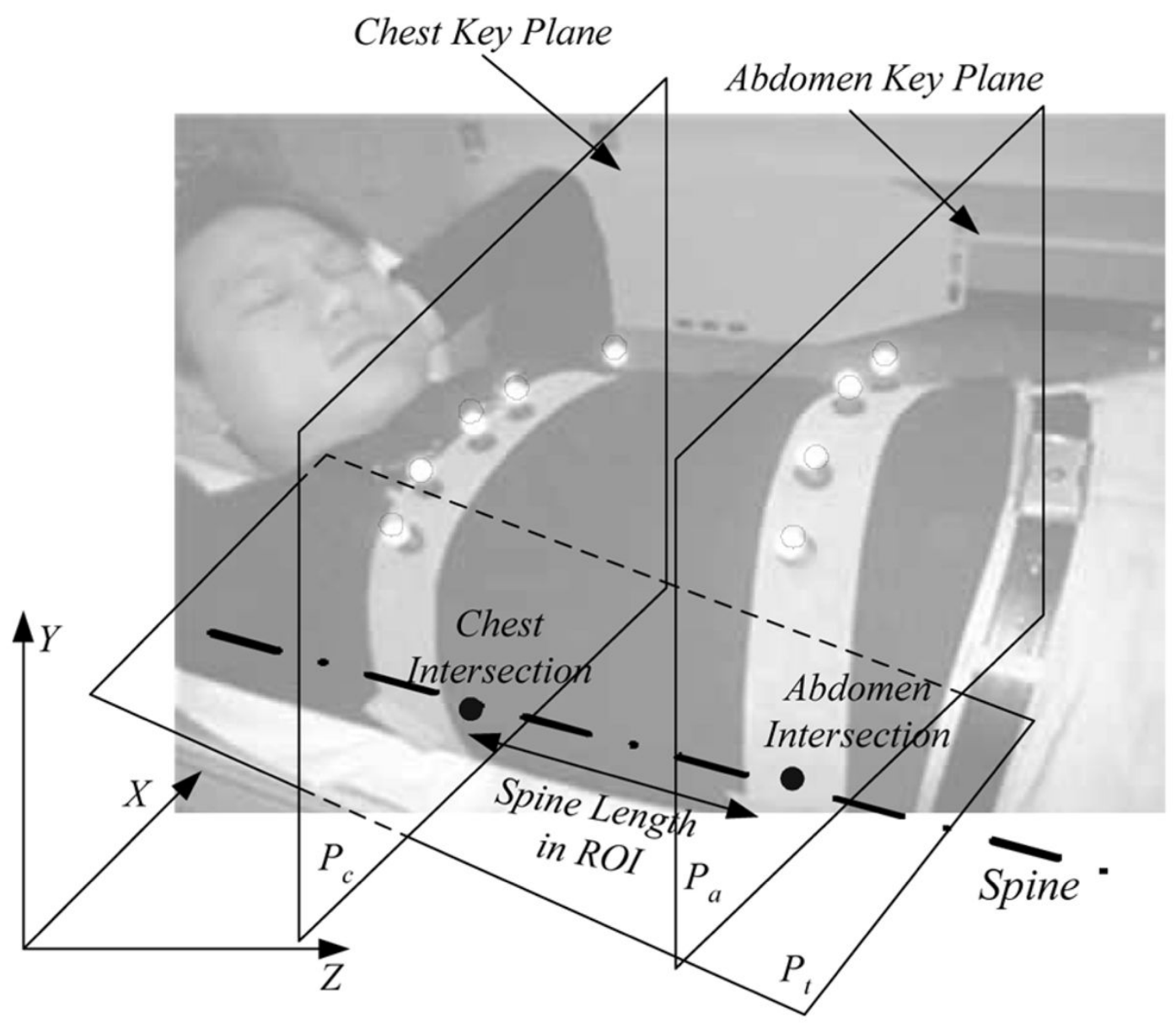

Fig. 7.

Body with belts showing table plane $P_{t}$, chest key plane $P_{c}$, abdomen key plane $P_{a}$, and the chest and abdomen key plane intersections with the spine. Shown also is the coordinate system employed with $x, y$, and $z$ as the lateral, vertical, and axial axes, respectively. 


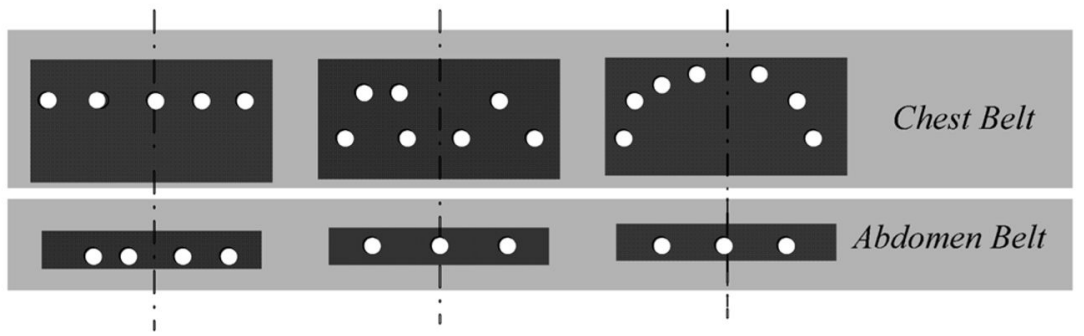

Fig. 8.

Sample patterns. 


\section{Reference Volume}

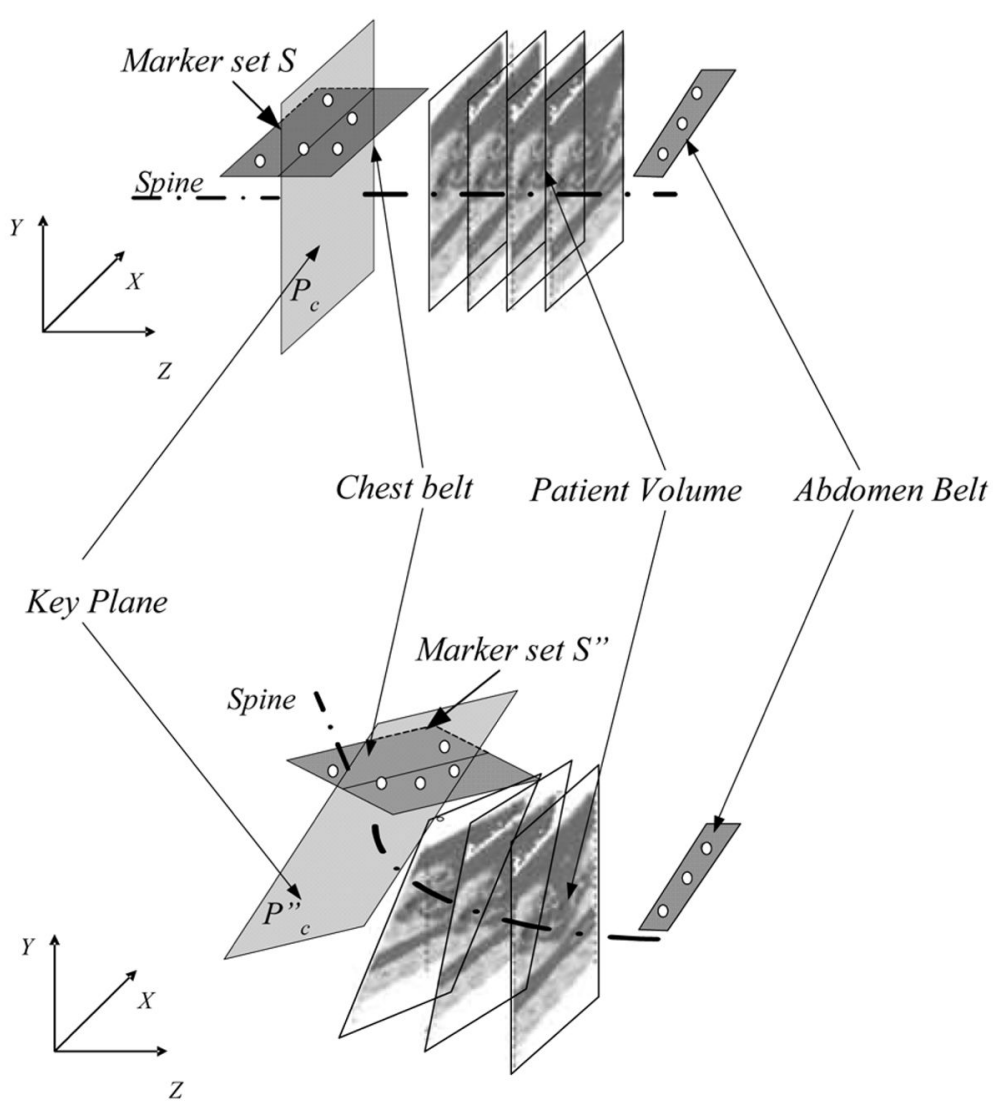

Deformed Volume

Fig. 9.

Chest key plane and chest belt. 


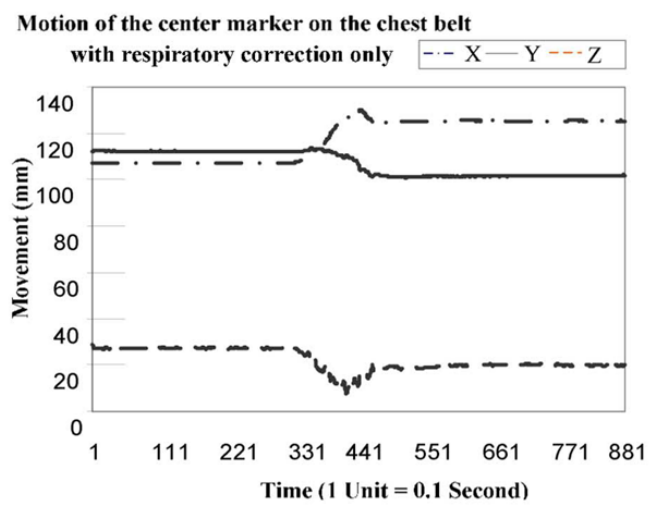

(a)

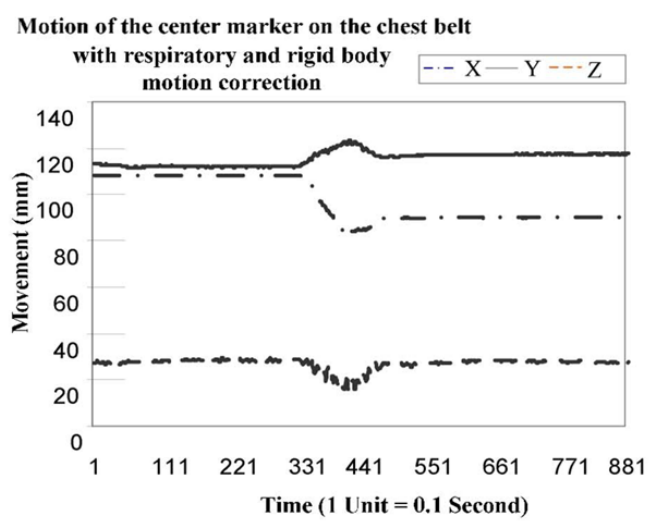

(b)

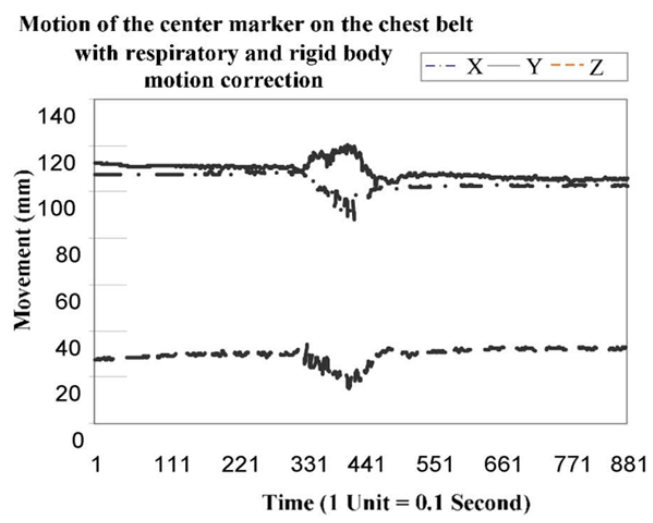

(c)

Fig. 10.

Comparison of the $x, y$, and $z$ locations of one marker on the chest belt before and after deformation correction. (a) Original tracked motion. (b) Motion after RBM correction. (c) Motion after rigid-body and deformation correction. Respiratory motion is small in amplitude for the chest marker compared to the other motion and was corrected in the original data. 


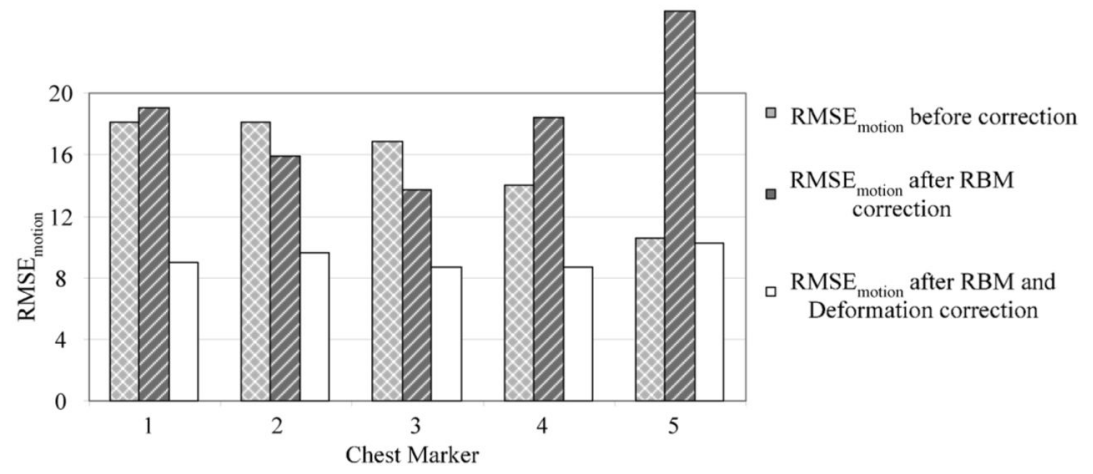

Fig. 11.

Comparison of $R M S_{\text {Emotion }}$ for each marker on the chest belt. 


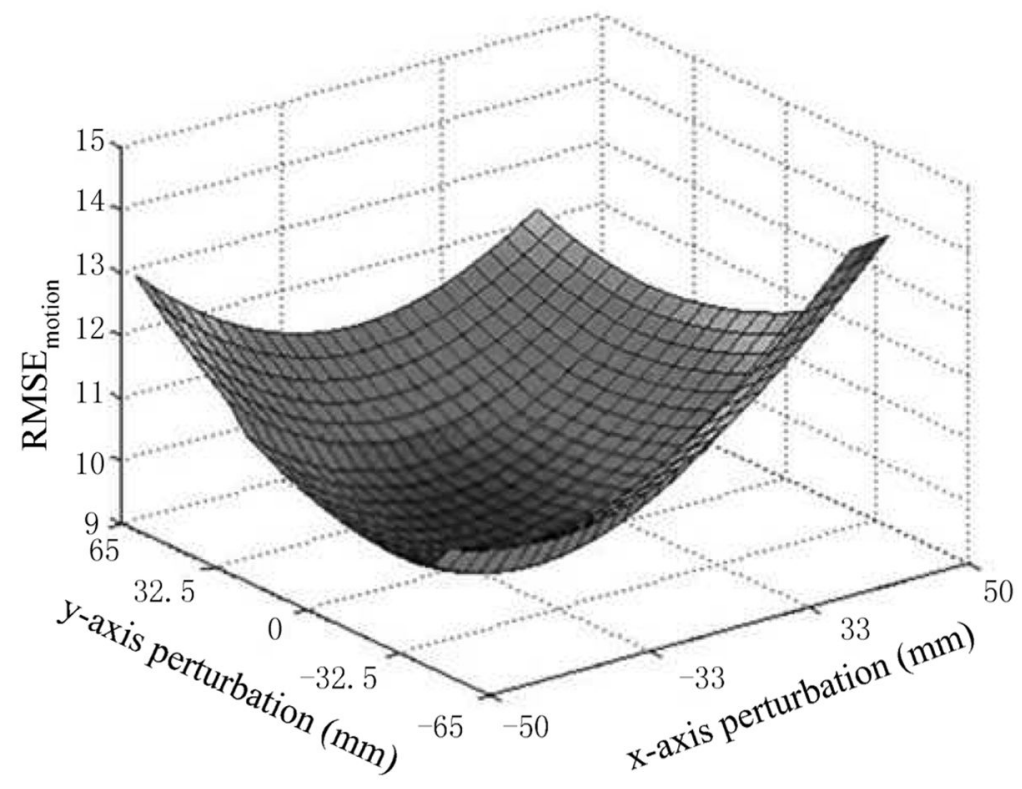

Fig. 12.

Distribution of $R M S_{\text {Erecon }}$ of one of the chest markers as a function of spine location in the $x y$-plane. 


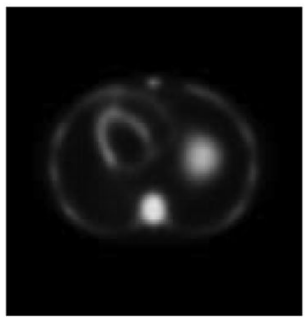

(a)

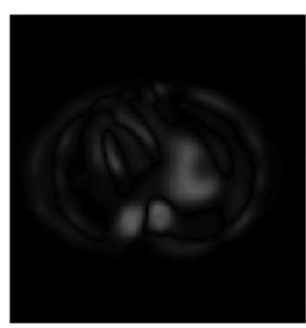

(d)

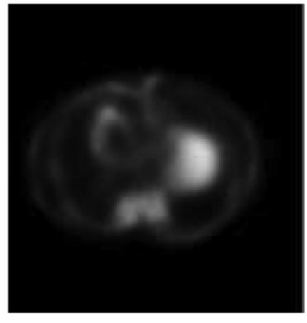

(b)

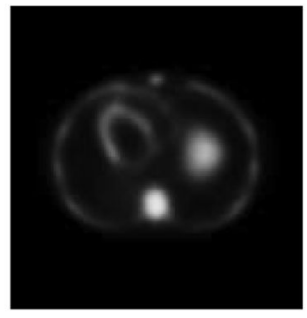

(c)

Fig. 13.

Comparison of transverse reconstruction slices from (a) MCAT projection set which did not include deformation, (b) MCAT projection set which included deformation but no reconstruction deformation correction, (c) MCAT projection set which included deformation and with deformation correction during reconstruction, (d) difference between (a) and (b), and (e) difference between (a) and (c). 
Sample coronal slices from the initial volume

Same positions from the 3D reconstruction volume with deformation but no correction
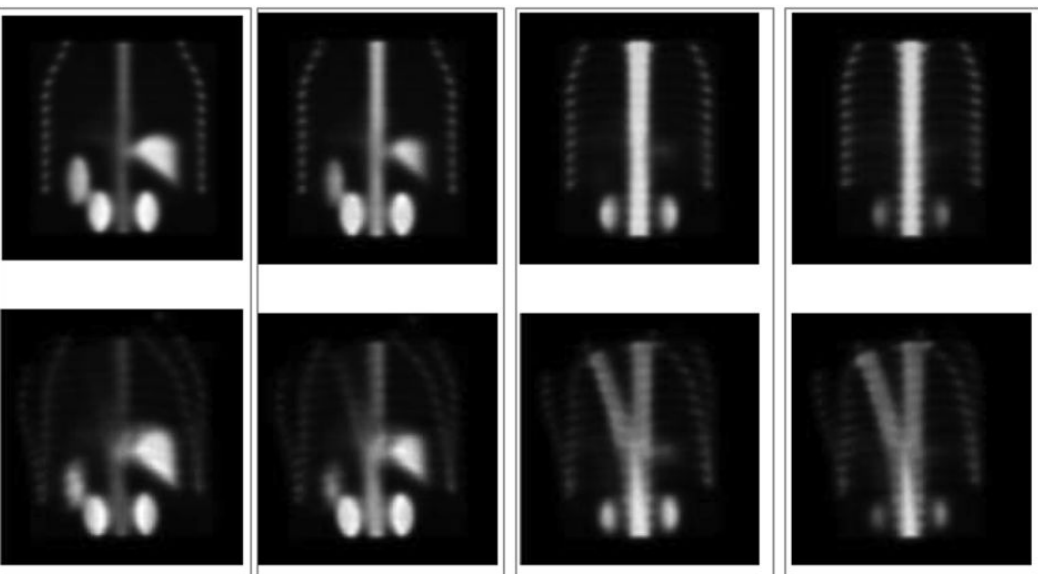

Same positions from the $3 \mathrm{D}$ reconstruction volume with deformation and correction
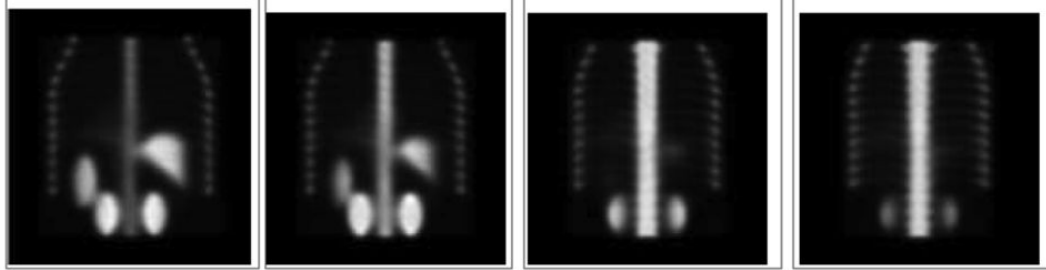

Fig. 14.

Coronal slices illustrating the impact of deformation and its correction on the spine, ribs, liver, spleen, and kidneys. Note the obvious bend of the spine laterally and its correction. Also note that the kidneys are not impacted by the bend or its correction as they were outside the ROI where it occurred. 


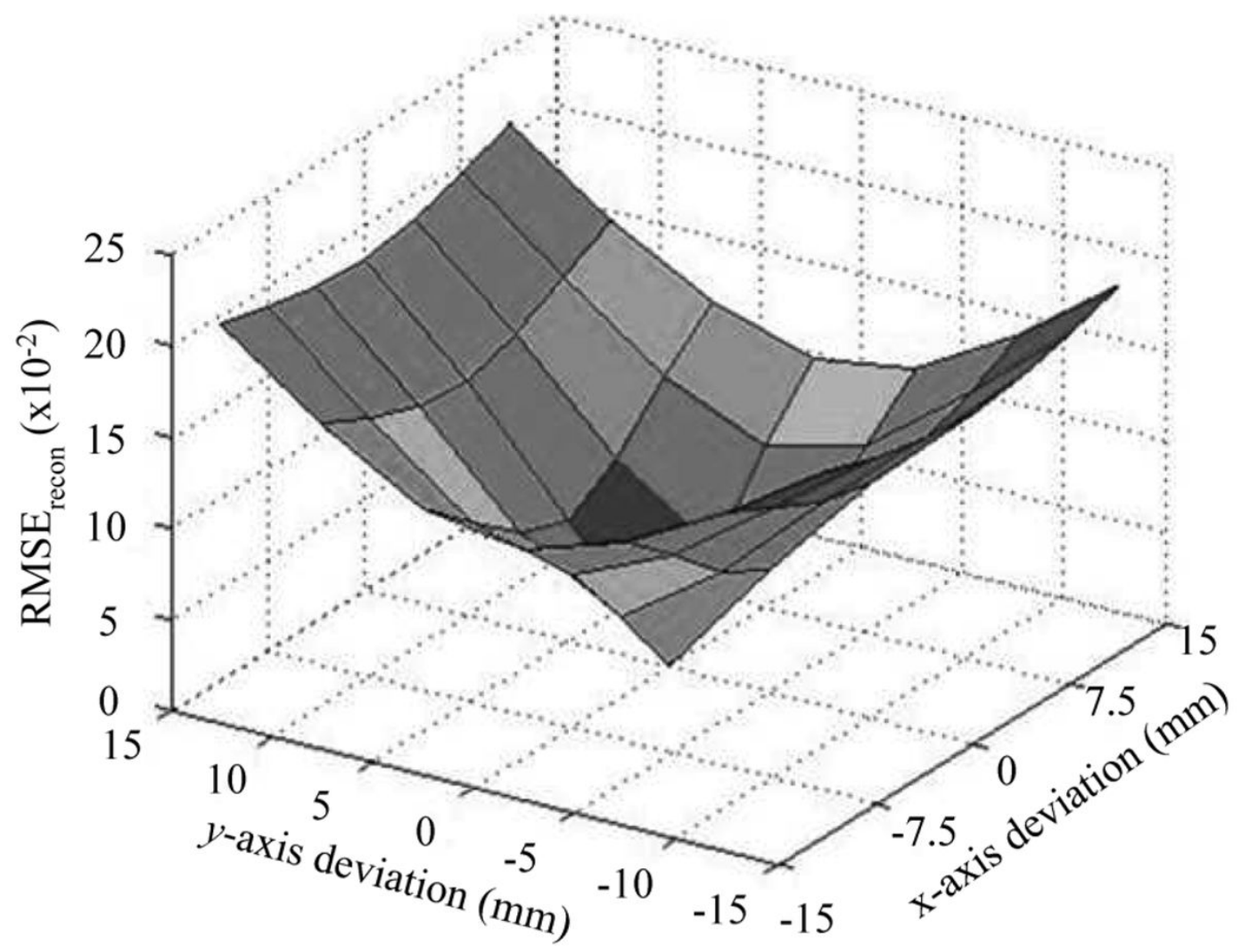

Fig. 15.

Distribution of $\mathrm{RMS}_{\text {Erecon }}$ of reconstructed volumes as a function of the spine locations in the $x y$ plane. 